\title{
The SinuSonic: reducing nasal congestion with acoustic vibration and oscillating expiratory pressure
}

This article was published in the following Dove Press journal:

Medical Devices: Evidence and Research

\author{
Alyssa Cairns' \\ Richard Bogan ${ }^{1-4}$ \\ 'Sleepmed, Inc., Columbia, SC, USA; \\ ${ }^{2}$ Bogan Sleep Consultants, LLC, \\ Columbia, SC, USA; ${ }^{3}$ The University of \\ South Carolina Medical School, \\ Columbia, SC, USA; ${ }^{4}$ The Medical \\ University of South Carolina, Charleston, \\ SC, USA
}

Introduction: Nasal congestion is common, burdensome, and costly. Current treatments are limited by partial/temporary relief and untoward side-effects. The goal of this study was to evaluate the performance of a novel, non-pharmacologic nasal device designed to reduce nasal congestion via simultaneous administration of acoustic vibration and gentle oscillating expiratory pressure.

Materials and methods: Patients were recruited from a tertiary care sleep clinic and all reported moderate-to-severe nasal congestion for $>2$ weeks $(\mathrm{N}=14 ; 64 \%$ female; $71 \%$ Caucasian). Visual analog scale (VAS) (10 items) quantifying nasal congestion and easeof-breathing were administered before and after SinuSonic application for 2-5 mins. Global and clinical impressions of change were assessed post-administration.

Results: Wilcoxon signed-rank tests indicated that post-test ranks were statistically improved from pre-test ranks for both VAS measures (congestion $p=0.002$; ease-of-breathing $p=0.003$ ). A binomial test indicated that the proportion of patients with $\geq$ minimal improvement on clinical and global impressions of change was higher than expected ( $100 \%$ vs expected $75 \%, p=0.018$ ).

Conclusion: Overall, outcomes were encouraging from this small pilot study with effect sizes in the moderate to large range and no reports of discomfort. It is probable that this device will provide acute, and possibly chronic, relief of nasal congestion with minimal side-effects.

Keywords: humming, sinus, congestion

\section{Introduction}

Nasal congestion, the uncomfortable sensation of nasal fullness, restriction, and/or draining, is a common phenomenon that plagues all of us at one time or another. Chronic or recurring nasal congestion, which affects between $10 \%$ and $20 \%$ of the global population, is a particularly troublesome experience associated with reduced quality of life, difficulty sleeping, reduced daytime performance, and increased health care utilization. ${ }^{1}$ It has been estimated that the financial impact of chronic nasal congestion is more than US \$5-10 billion annually. ${ }^{2,3}$

The four pairs of air-filled paranasal sinuses have an anatomically unfavorable position in that they lie in proximity to the nasal cavity which is heavily colonized by bacteria, viruses, mold, and other environmental pathogens. ${ }^{4}$ Inflammation of the nasal mucosa can cause obstruction of the ostia, the small openings that connect the sinuses and nasal cavity, causing poor sinus ventilation, inadequate drainage, discomfort, and even infection. ${ }^{5}$ A myriad of products exist to manage the symptoms of nasal congestion, including pain relievers and anti-inflammatories, decongestants, antihistamines, and nasal irrigation. However, these treatments are limited in that
Correspondence: Alyssa Cairns SleepMed, Inc., 700 Gervais Street, Suite 200, Columbia, SC 2920I, USA Tel + I 8037795454

Email acairns@sleepmedinc.com 
they provide only partial and/or temporary relief and sometimes have untoward side-effects. For example, it is well established that the use of nasal spray decongestants, such as oxymetazoline, can lead to a phenomenon known as rebound congestion or nasal spray dependence (Ramey et al, 2006). Additionally, systemic decongestant agents, such as pseudoephedrine, have been shown to increase blood pressure and are associated with dependency (DEA Title VII of Public Law 109-177). ${ }^{6}$ Given the limitations of current treatment options for nasal congestion, there is a need for novel non-pharmacologic therapies, ideally ones that target the unique anatomy of the sinus system.

The objective of this study was to evaluate the performance of a novel self-applied nasal device designed to reduce nasal congestion via simultaneous administration of acoustic vibration and gentle oscillating expiratory pressure. The SinuSonic consists of a fully disposable medicalgrade silicone nosepiece mounted to a resin body. The device is equipped with a flutter valve located at the top of the device which creates gentle, self-guided oscillating expiratory resistance. Acoustic vibration is emitted via a single circuit board speaker at the base of the device at approximately $128 \mathrm{~Hz}^{7} @ 80$ decibels (Figure 1).

The fundamental underpinnings of the device's design are based on research substantiating the therapeutic effects of positive expiratory pressure (PEP) and vibration on airway patency and mucous movement in chronic lung conditions. Although the SinuSonic device combines PEP and vibration differently than devices used for lung conditions (ie, by adding acoustic and mechanical vibration to PEP), we hypothesize that utilization of the aforementioned techniques will aid in upper airway patency and mucous movement, similar to the proven therapeutic effects for lower airway congestion. For example, a 2008 systematic review by Hristara-Papadopoulou et al found that devices that combine PEP and vibration produce statistically significant increase in lung volumes, ciliary motility, and sputum production for those with cystic fibrosis. ${ }^{8}$ The rationale for incorporating acoustic vibration is based on research suggesting that human humming improves sinonasal patency via increases in nitric oxide, ${ }^{4,9-11}$ a smooth muscle relaxant that has anti-inflammatory and antimicrobial/fungal/viral properties. ${ }^{12}$

\section{Materials and methods Participants}

A total of 14 adult participants were recruited from a private sleep disorders center. All patients were under the care of a sleep medicine specialist for a sleep condition, such as insomnia and sleep apnea but also complained of regular, daily nasal congestion for at least 2 weeks. Nasal congestion was corroborated with a Congestion Quantifier Test (COG) scores $>7 .^{13}$ Exclusion criteria included acute or chronic rhinosinusitis, severe mechanical obstruction (eg, complete unilateral deviated septum, polyps, etc.), pregnancy, or current decongestant use. A physical exam of the nasal passages was completed to ensure the absence of severe mechanical obstruction. Table 1 presents sample demographics. The study protocol was approved by Advarra, a central institutional review board, for the protection of human subjects. All subjects provided written informed consent and the study was conducted in accordance with the Declaration of Helsinki. As part of the informed consent process, subjects were apprised of the

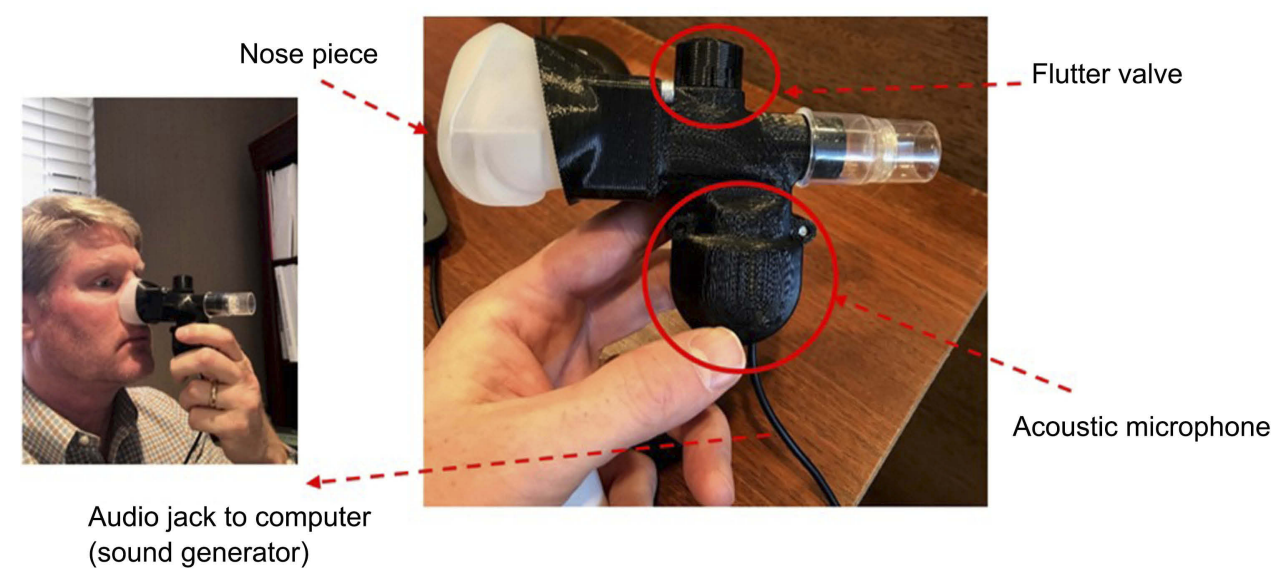

Figure I The SinuSonic device. Depiction of the SinuSonic nasal device. The individual depicted here was not a research subject and provided written consent for this image to be published. 
Table I Demographics

\begin{tabular}{|l|l|}
\hline Sample size & $\mathbf{N}=\mathbf{I 4}$ \\
\hline Sex (\% female) & $64 \%(\mathrm{n}=9)$ \\
Race (\% Caucasian) & $71 \%(\mathrm{n}=10)$ \\
Age (years) (min-max) & $52.1(30-7 I)$ \\
BMI (kg/m²) & $32.1(19.9-46.1)$ \\
COG-7 (min-max) & $18.3(13-2)]$ \\
\hline
\end{tabular}

Abbreviations: COG-7, Congestion Quantifier Test; BMI, body mass index.

principal investigator's (PI; Bogan) scientific and commercial involvement with the Sinusonic.

\section{Procedure}

A research coordinator administered written consent, screened the patient for inclusion and administered the COG scale. If inclusion criteria were met, the patient underwent a brief examination by a study physician, including a nasal cavity examination and clinical assessment of nasal congestion severity. Next, the study coordinator administered two 10-item paper-based visual analog scale (VAS) aimed as assessing nasal congestion and ease-of-breathing (Figure 2). Each patient was instructed to circle the numeric value that corresponded to their symptom severity on the VAS scales prior to treatment. The patient was then instructed to self-apply the SinuSonic nasal mask and "breathe naturally, but with increased exhalation force [until the flutter sound is heard] for 2 to 5 mins, based on comfort". After device use, the patient was administered two post-VAS scales (identical to the pre-VAS measures) and instructed to circle the numeric value that corresponded to their symptoms. Next, patients rated their symptom change on a 7-item Likert Patient Global Impression of Change (PGI). The PGI was measured on the following scale: $1=$ very much better; $2=$ much better; $3=$ a little better; $4=$ no change; $5=$ a little worse; $6=$ much worse; $7=$ very much worse. Finally, the same physician that conducted the baseline assessment completed a Clinical Global Impression of Change (CGI). The CGI was formatted to the same Likert scale as the PGI $(1=$ very much better; $2=$ much better; $3=$ a little better; $4=$ no change; $5=$ a little worse; $6=$ much worse; $7=$ very much worse). All physicians were blinded to the responses from the patient's self-assessment of change (PGI) to allow independent observation. In some cases, the PI of the study (who has financial interest in the study) completed the assessments.

\section{Statistical analyses}

Outcome variables for analysis included the difference score between pre- and post-VAS scales and the patient and clinical global impressions of change. Analyses were completed by a researcher with no commercial interest in the Sinusonic. Demographic analyses and non-parametric tests were performed with SPSS 23.0 (SPSS Inc., Chicago, IL, USA). Pre-post differences in VAS scores were assessed

A Visual analog scale for nasal congestion

Please rate your level of nasal congestion below

\begin{tabular}{|c|c|c|c|c|c|c|c|c|c|}
\hline 1 & 2 & 3 & 4 & 5 & 6 & 7 & 8 & 9 & 10 \\
\hline No o & stion & & \multicolumn{5}{|c|}{ Moderate congestion } & \multicolumn{2}{|c|}{ Worst congestior } \\
\hline
\end{tabular}

B Visual analog scale for ease-of-breathing

Please rate your level of difficulty to breathe through your nose

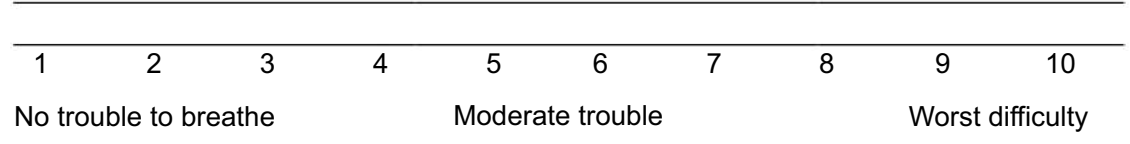

Figure 2 Visual analog scale for nasal congestion (A) and visual analog scale for ease-of-breathing (B). Self-assessment measure of subjective nasal congestion and ease-ofbreathing. This measure was used before and after Sinusonic, and the difference score pre-post was tabulated to analyze symptom change. 
with Wilcoxon rank-sum analysis. Global Impressions of Change (CGI/PGI) were assessed with a one-sample Binomial test of the hypothesis that $\geq 75 \%$ of the sample would achieve " $\geq$ minimal improvement" (based on expert consensus). All comparisons were one-tailed and significance was set at the 0.05 level.

\section{Results}

As Table 1 illustrates, most of the sample was comprised of females (64\%) and those identified as Caucasian (71\%). All subjects reported severe nasal congestion (mean $\mathrm{COG}=18.3) .{ }^{13}$ All outcome measures were encouraging for this small pilot study of a single application of the SinuSonic in a clinic environment. Wilcoxon signed-rank tests indicated that post-test ranks were statistically improved from pre-test ranks for both VAS measures (congestion $=\mathrm{Z}=3.1, p=0.002$; ease-of-breathing $=\mathrm{Z}=2.9, p=0.003$ ). As Figure 3 illustrates, all patients reported improvement in nasal congestion after a single application of the SinuSonic. Likewise, all but one subject reported improved breathing after Sinusonic use. A binomial test indicated that the proportion of patients with at least minimal improvement on CGI/PGI was higher than expected $(100 \%$ actual vs expected $75 \%$ for both clinician and self-assessed, $p=0.018$; Figure 4). Importantly, no subjects reported a worsening of symptoms or discomfort from SinuSonic use.

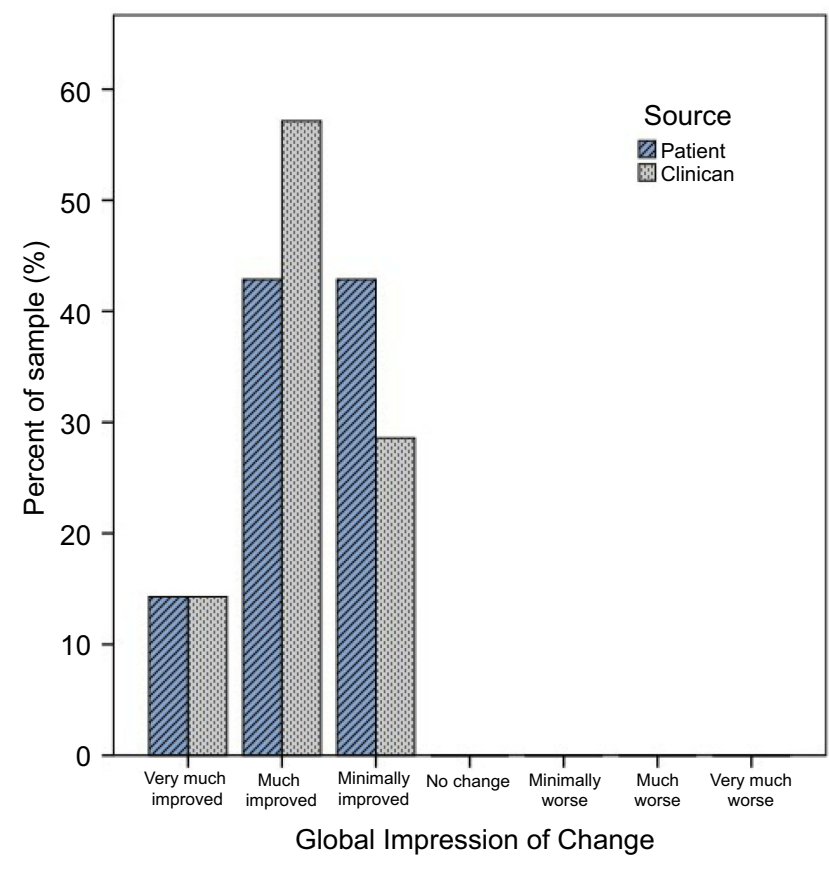

Figure 4 Clinical and patient global impressions of change. Binomial test - the proportion of patients with $\geq$ minimal improvement on CGI/PGI was higher than expected ( $100 \%$ vs expected $75 \%$ for both clinician and self-assessed, $p=0.018$ ). Abbreviation: CGI/PGI, Clinical Global Impressions of Change/Patient Global Impression of Change.

\section{Discussion and conclusion}

This was a simple, proof-of-concept study in a small sample of treatment-seeking sleep clinic patients with self-reported chronic nasal congestion. Results from this were generally

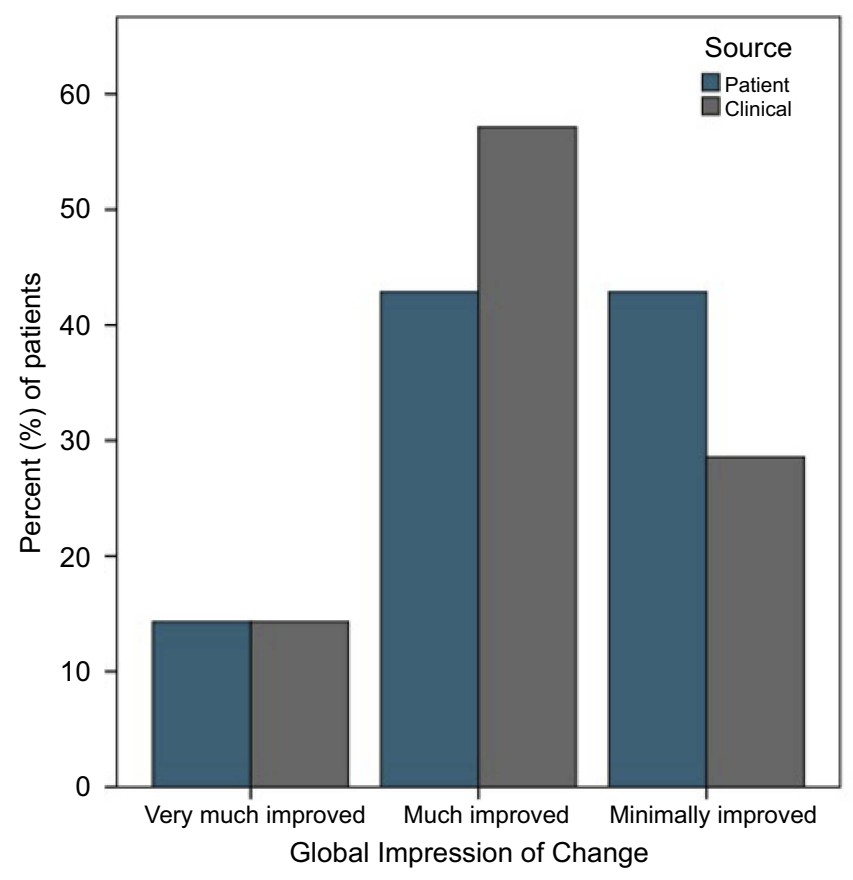

Figure 3 Improvement in self-reported breathing and congestion. X-axis represents the difference score between VAS scales pre- vs post-treatment. Wilcoxon signed-rank test revealed VAS post-test ranks were statistically improved over pre-test ranks (VAS congestion $Z=3.1, p=0.002 ;$ VAS breathing $Z=2.9$, $p=0.003$ ).

Abbreviation: VAS, visual analog scale. 
consistent with the body of literature supporting vibration and PEP on lower airway patency. ${ }^{8}$ That is, these limited data suggested that a single exposure to the SinuSonic in a controlled clinic environment provided short-term relief from nasal congestion symptoms with effect size in the mediumto-large range and minimal side-effects. The finding that both clinical and patient global impressions of change convergently validated self-reported symptom change was encouraging considering the absence of placebo control and small sample size.

This study had several limitations. Most obviously, this study was small, did not employ a placebo control, and observers and patients were not blinded. Thus, it is likely that some of the effects observed in this study were due to placebo effect and observer bias. Another limitation was variable therapy exposure in that patients were instructed to terminate the SinuSonic at-will. It is thus unknown the therapeutic "dose" of the SinuSonic. Despite these limitations, these data support the rationale for a larger-scaled study which will employ a sham control, more robust measures of nasal airflow change, and longer follow-up.

\section{Abbreviations}

COG, Congestion Quantifier Test; PGI, Patient Global Impression of Change; CGI, Clinical Global Impression of Change.

\section{Acknowledgment}

The researchers warmly thank the participants of this study. This study was funded by Bogan Sleep Consultants, LLC.

\section{Author contributions}

$\mathrm{AC}$ - data analysis, data interpretation, and full manuscript drafting. RB - conception and design of the study and data interpretation. All authors contributed to data analysis, drafting and revising the article, gave final approval of the version to be published, and agree to be accountable for all aspects of the work.

\section{Disclosure}

Drs Bogan and Cairns are employed by SleepMed, Inc., a for-profit sleep diagnostic, therapy, and research company.
Dr Bogan is a consultant for Jazz Pharmaceuticals and is on the speaker's board for Jazz Pharmaceuticals. Dr Bogan is a shareholder in SinuSonic and owner of Bogan Sleep Consultants, LLC. Dr Bogan also reports grants, and received non-financial support from Healthy Humming, LLC, outside the submitted work; in addition, Dr Bogan has a patent 0141-0001CIP pending to Healthy Humming, LLC. Dr Cairns reports personal fees from Sinusonic, LLC, during the conduct of the study and grants from Jazz Pharmaceuticals, outside the submitted work. The authors report no other conflicts of interest in this work.

\section{References}

1. Stewart M, Ferguson B, Fromer L. Epidemiology and burden of nasal congestion. Int J Gen Med. 2010;3:37-45.

2. Meltzer EO, Bukstein DA. The economic impact of allergic rhinitis and current guidelines for treatment. Ann Allergy Asthma Immunol. 2011;106(2 Suppl):S12-S16. doi:10.1016/j.anai.2010.10.014

3. DeConde AS, Soler ZM. Chronic rhinosinusitis: epidemiology and burden of disease. Am J Rhinol Allergy. 2016;30(2):134-139. doi:10.2500/ajra.2016.30.4297

4. Lundberg JO. Nitric oxide and the paranasal sinuses. Anat Rec (Hoboken). 2008;291(11):1479-1484. doi:10.1002/ar.20782

5. Wagenmann M, Naclerio RM. Anatomic and physiologic considerations in sinusitis. J Allergy Clin Immunol. 1992;90:419-423. doi:10.1016/0091-6749(92)90161-T

6. Salerno SM, Jackson JL, Berbano EP. Effect of oral pseudoephedrine on blood pressure and heart rate: a meta-analysis. Arch Intern Med. 2005;165(15):1686-1694. doi:10.1001/archinte.165.15.1686

7. Durand M, Le Guellec S, Pourchez J, et al. Sonic aerosol therapy to target maxillary sinuses. Eur Ann Otorhinolaryngol Head Neck Dis. 2012;129(5):244-250. doi:10.1016/j.anorl.2011.09.002

8. Hristara-Papadopoulou A, Tsanakas J, Diomou G, Papadopoulou O. Current devices of respiratory physiotherapy. Hippokratia. 2008;12 (4):211-220.

9. Weitzberg E, Lundberg JO. Humming greatly increases nasal nitric oxide. Am J Respir Crit Care Med. 2002;166(2):144-145. doi:10.1164/rcem.200202-138BC

10. Jain B, Rubinstein I, Robbins RA, Leise KL, Sisson JH. Modulation of airway epithelial cell ciliary beat frequency by nitric oxide. Biochem Biophys Res Commun. 1993;191(1):83-88. doi:10.1006/bbrc.1993.1187

11. Maniscalco M, Weitzberg E, Sundberg J, Sofia M, Lundberg JO. Assessment of nasal and sinus nitric oxide output using single-breath humming exhalations. Eur Respir J. 2003;22(2):323-329.

12. Rosselli M, Keller PJ, Dubey RK. Role of nitric oxide in the biology, physiology and pathophysiology of reproduction. Hum Reprod Update. 1998;4(1):3-24. doi:10.1093/humupd/4.1.3

13. Stull DE, Krouse J, Meltzer EO, et al. Development and validation of the Congestion Quantifier seven-item test (CQ7): a screening tool for nasal congestion. Value Health. 2007;10(6):457-465. doi:10.1111/ j.1524-4733.2007.00201.x 


\section{Publish your work in this journal}

Medical Devices: Evidence and Research is an international, peerreviewed, open access journal that focuses on the evidence, technology, research, and expert opinion supporting the use and application of medical devices in the diagnosis, monitoring, treatment and management of clinical conditions and physiological processes. The identification of novel devices and optimal use of existing devices which will lead to improved clinical outcomes and more effective patient management and safety is a key feature of the journal The manuscript management system is completely online and includes a very quick and fair peer-review system. Visit http:// www.dovepress.com/testimonials.php to read real quotes from published authors.

Submit your manuscript here: https://www.dovepress.com/medical-devices-evidence-and-research-journal 